\title{
Investigation of an Arc-induced Long Period Fiber Grating Inscribed in a Photonic Crystal Fiber with Two Large Air Holes
}

\author{
Sunduck Kim, Gil Hwan Kim, Kyu-Jin Hwang, Sun Do Lim, Kwanil Lee, Sang Hyuck Kim, \\ and Sang Bae Lee* \\ Photonics Research Laboratory, Division of Intelligent System, Korea Institute of Science and \\ Technology (KIST), Seoul 130-791, Korea \\ Je-Myung Jeong \\ Optical Communication Laboratory, Division of Electrical and Computer Engineering, \\ Hanyang University, Seoul 133-791, Korea
}

(Received September 30, 2009 : revised November 11, 2009 : accepted November 12, 2009)

\begin{abstract}
A photonic crystal fiber with two large air holes outside the holey cladding region is fabricated to induce an effective long periodic grating (LPG) in the core by an electric arc discharge. We believe that the two large air holes lead to the asymmetric perturbation in the core under the electric arc discharge, thereby introducing the coupling to the first higher-order mode. The transmission characteristics of the PCF with the LPG for the external perturbation such as strain, curvature, and temperature are also investigated. It was found that the shift of resonance peak in the transmission spectrum depends on the bending direction. The curvature of $8.55 \mathrm{~m}^{-1}$ results in the center wavelength shifts of $1.8,4.3$, and $11 \mathrm{~nm}$ for a vertical, diagonal, and horizontal direction of the curvature to the large air-hole alignment, respectively.
\end{abstract}

Keywords: Long period fiber grating, Photonic crystal fiber, Sensor

OCIS codes : (050.2770) Gratings; (060.0060) Fiber optics and optical communications; (060.5295) Photonic crystal fibers

\section{INTRODUCTION}

Long period fiber grating (LPFG) has drawn much attention in the development of optical devices $[1,2]$ and sensors [3]. In particular, the LPFGs are of great interest in sensorapplications because the resonance condition is very sensitive to various measurands: strain, curvature, external refractive index, and temperature. Recently, the LPFGs written in an all-silica photonic crystal fiber (PCF) [4] have been reported, and it turned out that the resonance condition, unlike those from the conventional fibers, is substantially insensitive to the temperature variation [5]. To this end, the PCF-based LPFG has been extensively studied forpotential applications in temperature insensitive optical sensors. To date, many efforts have been made to write the LPG in the PCFs, with a $\mathrm{CO}_{2}$ laser [6], a femtosecond laser [7], and an arc discharge [9] as well as mechanical pressure [8]. These methods give rise to periodic deformation in the core of the PCF. However, it is in

*Corresponding author: sblee@kist.re.kr practicehard to introduce the deformations in thecore by external perturbations because the PCF consists of a single material and the core is surrounded by the air holes. Moreover, the PCFs typically with hexagonal structure have circular symmetry in the air-hole lattice, thereby making it even harder to fabricate the LPFG coupling between relevant anti-symmetric modes.

In this work, we fabricated a PCF with two large air holes insidethe outer cladding, in which an asymmetrical stress distribution to the core can be effectively induced by an electric arc discharge. We also investigated the transmission characteristics of the PCF with the LPG under the strain, curvature, and temperature variations. The experimental results are well compared with the theoretical results.

\section{FABRICATION OF THE LPFG IN THE PS-PCF}

Figure 1 shows the scanning electron microscope (SEM) image of the PCF which is composed of two 
large circular air holes in the outside of cladding region like a panda-shaped fiber. So we name it panda-shaped PCF (PS-PCF). The PS-PCF was fabricated with the stack-and-draw process in our laboratory [10, 11]. The small air holes in the inner cladding and two large air holes in the outer cladding region are $3.74 \mu \mathrm{m}$ and $15.87 \mu \mathrm{m}$ in diameter, respectively. Furthermore, the ratio of small air hole diameter to pitch is 0.6 , which means PS-PCF becomes a multi-mode fiber. In this case, higher modes can interact with the fundamental core mode. The polarization dependence loss (PDL) and differential group delay (DGD) were measured to be $0.2 \mathrm{~dB}$ and $0.05 \mathrm{ps} / \mathrm{m}$, respectively.

In LPFGs of a multi-mode fiber, the phase matching condition, between the propagations of the fundamental core and higher modes at the wavelength $\lambda$ can be applied in the same way in the single-mode fiber the core mode couples with cladding modes. The phase matching condition can be expressed by

$$
\lambda^{m}=\left[n_{\text {core }}(\lambda)-n_{\text {core }}{ }^{m}(\lambda)\right] \Lambda
$$

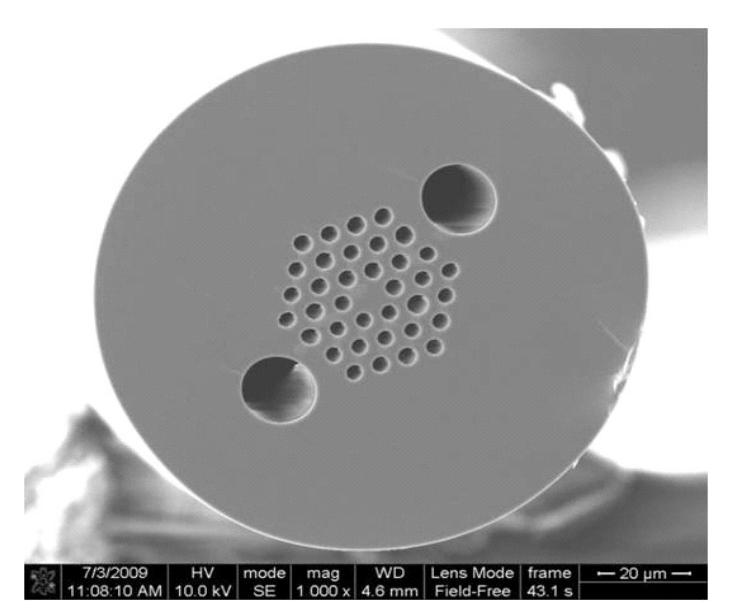

FIG. 1. SEM image of the fabricated PS-PCF.

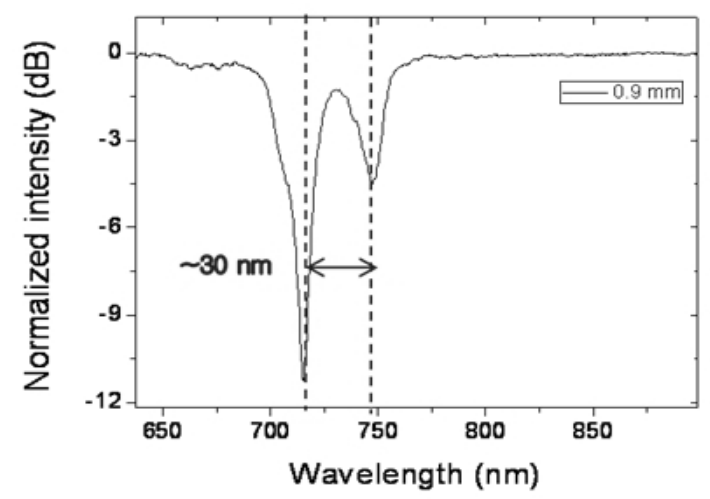

FIG. 2. Transmission spectra of the LPFG with the grating period of $900 \mu \mathrm{m}$ generated by the periodically grooved plate. where $n_{\text {core }}(\lambda)$ is the effective refractive index of the propagating core mode at wavelength $\lambda, n_{\text {core }}{ }^{m}(\lambda)$ is the refractive index of the $m$-th core mode in the multimode fiber, and $\Lambda$ is the period of the LPFG.

When we apply the mechanical pressure using a $\mathrm{V}$-groove, two separated coupling modes, whose wavelength spacing is around $30 \mathrm{~nm}$, were observed as shown in FIG. 2. Thus in order to indentify these coupling modes, first we changed the center wavelength of the resonance peaks of the LPFG by adjusting the period of the V-groove, then matched it to the center wavelength of a helium-neon laser. After the adjustment, a setup for the confirmation was built as shown in FIG. 3 (a). We could see the far field pattern of the higher order modes as shown

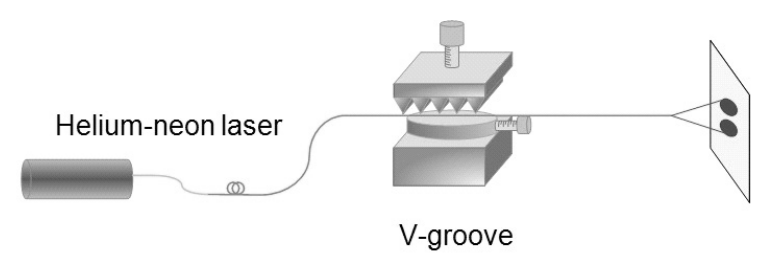

(a)

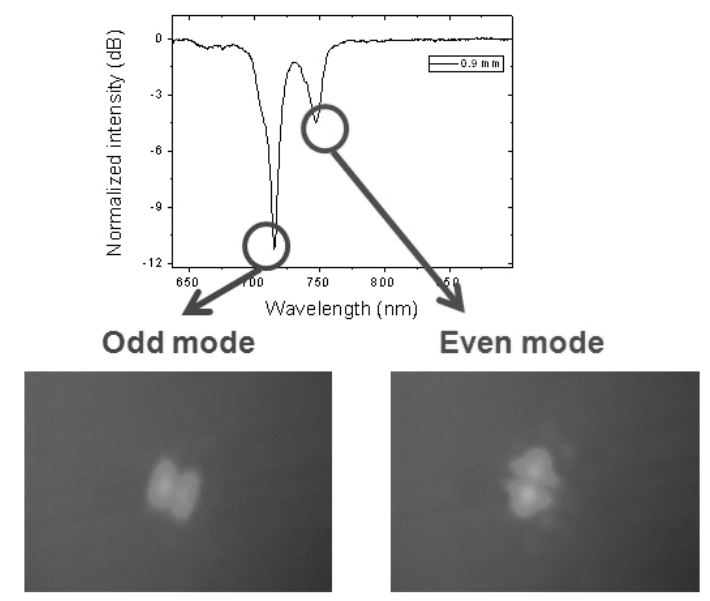

(b)
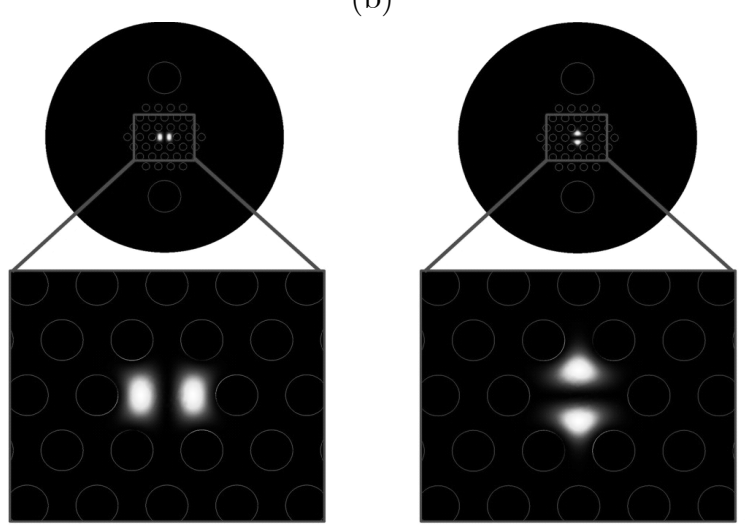

(c)

FIG. 3. (a) Experimental setup for the verification of the coupling mode using a V-groove with the period of $900 \mu \mathrm{m}$, (b) far mode field pictures with respect to coupling modes, and (c) calculation results of the coupling mode. 
in FIG. 3 (b). In addition, calculations were done for the verification of the modes using $2 \mathrm{D}$ finite-element simulation software, Multiphysics. The first coupling mode is the even mode, and second mode is the odd mode of LP11. This can be attributed to any slight disorder in the inner air-hole lattice [12].

With the results from the mechanical pressure method for the grating period, the LPFG used in this paper was fabricated with the experimental setup as shown in FIG. 4. (a). A commercial splicer, Fujikura FSM-40F was used to provide an electric-arc current with specific intensity and expose time. In the experiment, the discharge intensity and time were 2 bit and $200 \mathrm{~ms}$, where a nominal power unit 'bit' is used in the Fujikura which is digitized analog arc current. In addition, constant tension was applied to the fiber to ensure that the fiber was straight by attaching a balance weight onto the fiber. While an arc discharge was periodically induced by a translator with a remote controller, the transmission spectrum of a light transmitted from a broadband light source was observed by an optical spectrum analyzer (OSA).

Figure $4(\mathrm{~b})$ shows the transmission spectra of the LPFG with a grating period of $900 \mu \mathrm{m}$. The total length and period number of the LPFG were $8.1 \mathrm{~mm}$ and 10 periods. The first higher coupling mode with the center wavelength of $727.4 \mathrm{~nm}$ was used for the experimental sensing of the measurands such as curvature,

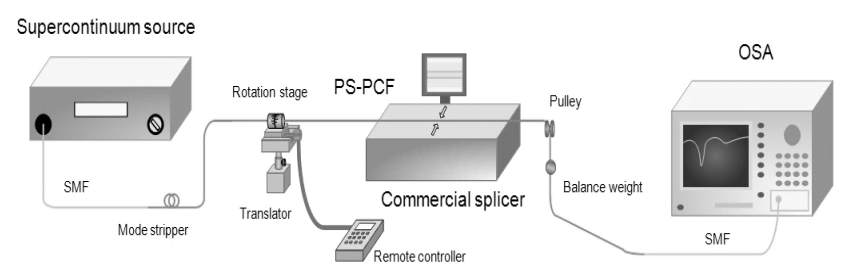

(a)

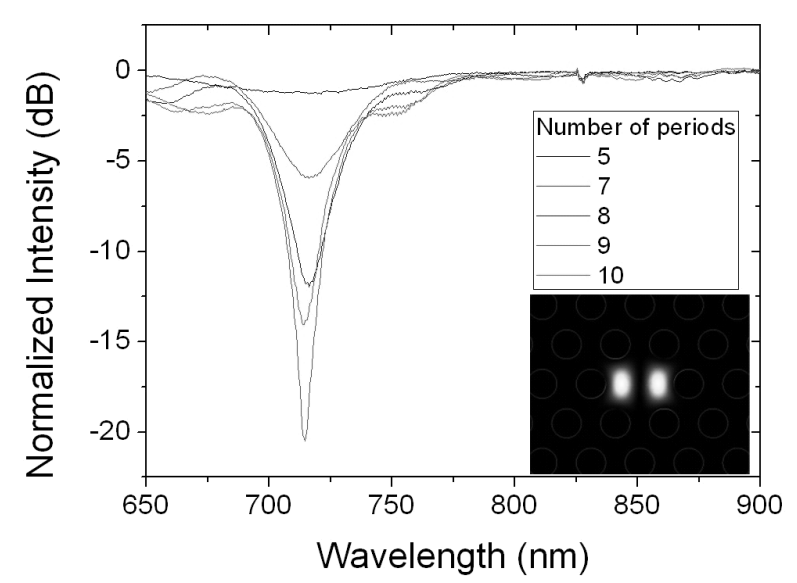

(b)

FIG. 4. (a) Experimental setup for the fabrication of LPFGs, and (b) transmission spectra of the LPFG with a grating period of $900 \mu \mathrm{m}$. strain, temperature.

Here, note that only one resonance peak remains. Even though the mode was originally supposed to be split into two modes, an even mode, and an odd mode, with some particular condition in the inner air-hole lattice, the mode can be almost coupled into one mode, here an odd mode. Because of the two large air holes in the fiber, the conditions were satisfied by imperfectly-distributed heat of an arc discharge onto the fiber.

\section{SENSITIVITIES TO CURVATURE, STRAIN, TEMPERATURE}

\section{Spectral curvature characteristic of the LPFG in the PS-PCF}

The experimental setup for measurement of curvature using the LPFG inscribed in PS-PCF is shown in FIG. 5 (a). Both ends of the 35-cmm long PS-PCF having the LPFG at the mid-point were clamped by two rotational stages. One of them was mounted on a translator. The bending was induced by moving the translator inwards. When the bending displacement at the center of the LPFG is $d$ and the

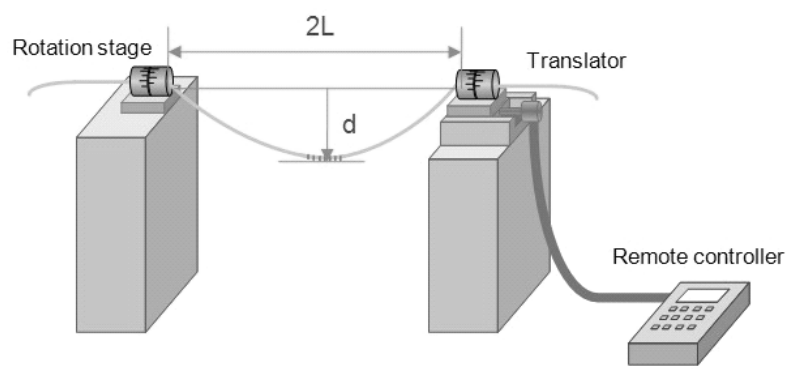

(a)

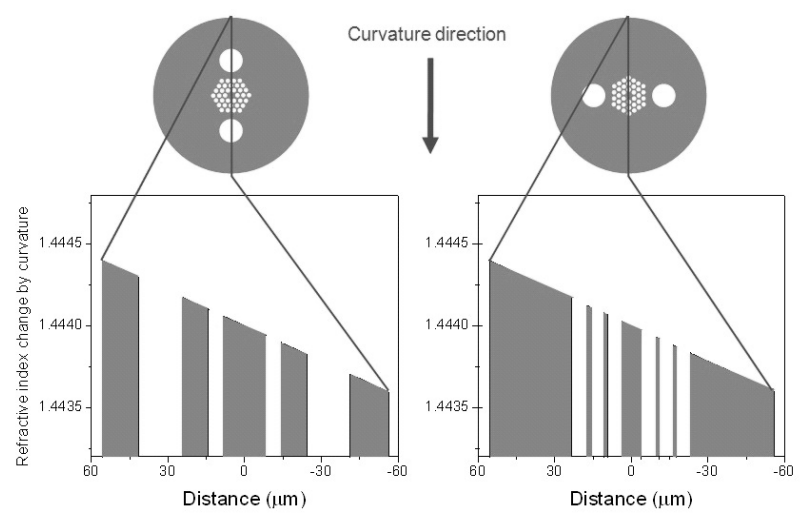

(b)

FIG. 5. (a) Experimental setup for the measurement of curvature, (b) the calculated results of reflective index distribution with the curvature of $5 \mathrm{~m}^{-}{ }^{1}$, when two large holes are aligned, one vertically and one horizontally to the axis of the curvature. 
half-distance between two clamps is $L$, the induced curvature is given by

$$
1 / R=2 d /\left(d^{2}+L^{2}\right)
$$

The calculation results [13] in FIG. 5 (b) show that when the curvature of $5 \mathrm{~m}^{-1}$ is applied horizontally and vertically to the axis of the two large air holes, in the core region, there is no difference between the distributions of the refractive indices in the two cases. Therefore, initially the curvature sensitivities do not depend on the axis of the bending. However, as explained previously, since the coupling mode propagates orthogonally to the axis of two large air holes, the curvature sensitivity will be maximized when the two large air holes are aligned horizontally to the axis of the curvature.

When curvature is applied to a fiber, the propagating higher core modes have the modified effective refractive index, which leads to changes in the center wavelength and depth of the resonance peaks. The origin of the curvature sensitivity may be understood by differentiating equation (1) [14].

$$
\frac{d \lambda^{m}}{d C}=\Lambda\left(\frac{d n_{\text {core }}}{d C}-\frac{d n_{\text {core }}{ }^{m}}{d C}\right)+\left(n_{\text {core }}-n_{\text {core }}{ }^{m}\right) \frac{d \Lambda}{d C}
$$

As the curvature increases, the change in the effective reflective index of the higher core mode is higher than the change in the effective reflective index of the core. Therefore the first term in Eq. (3) for the material contribution is dominant, while the second term in Eq. (3) for the waveguide contribution can be ignored under the assumption that the core is located exactly at the center.

The transmission characteristics of the LPFG inscribed in the PS-PCF are shown in FIG. 6 as a function of bending curvature. The center wavelength of the resonance peak is shifted to the longer wavelength direction as the bending curvature increases regardless of the alignment of the two large air holes. Since the variation of the refractive index of the second core mode is higher than that of the fundamental core mode, the center wavelength of the resonance peak is shifted in one direction in accordance with the order of the higher core mode which can be a blue or a red shift. The amount of the center wavelength shift was observed to depend upon the direction of the curvature. When the bending direction is perpendicular to the two large air holes, the maximum sensitivity occurred in accordance with our expectation.

The sensitivities to the curvature for the different orientation of the curvature direction with respect to the two large air holes are shown in FIG. 7. The curvature sensitivity with two large air holes placed vertically with respect to the curvature direction was

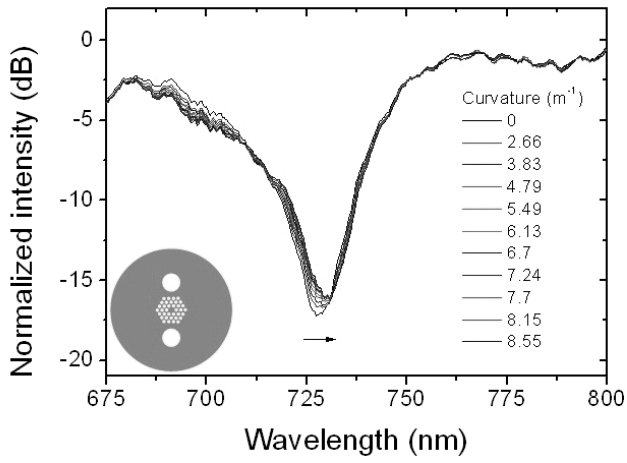

(a)

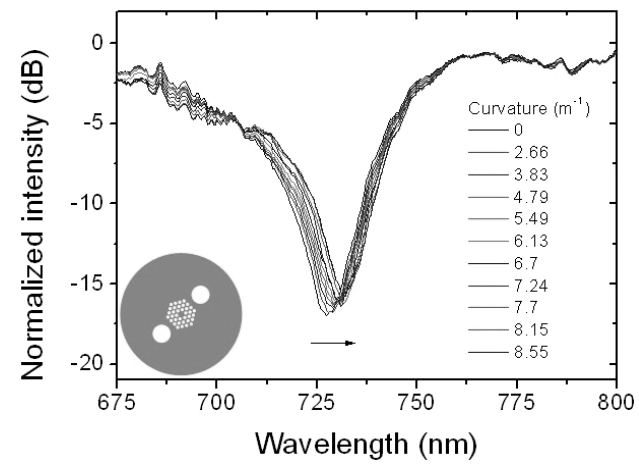

(b)

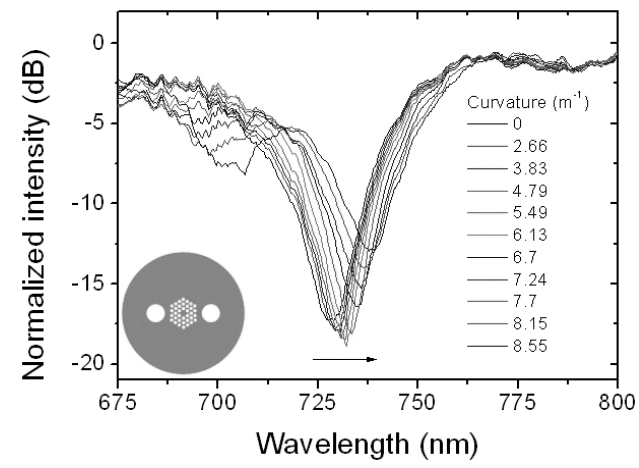

(c)

FIG. 6. Transmission spectra of the LPFG with the bending curvature change when two large air holes are placed (a) vertically, (b) diagonally, and (c) horizontally.

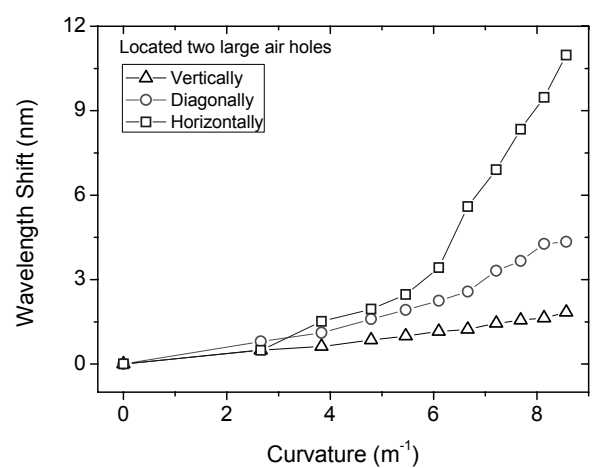

FIG. 7. Variation of the resonance peak with respect to three different positions of the two large air holes. 
the highest, whereas the sensitivity was the lowest with horizontally aligned holes. When the curvature was 8.55 $\mathrm{m}^{-1}$, the center wavelength shifts were measured to be $1.8,4.3,11 \mathrm{~nm}$ in when the two large air holes were aligned vertically, diagonally, horizontally, respectively.

The dependence of the sensitivity on the orientation of curvature is attributed to the propagating coupling mode by the LPFG in the PS-PCF. That is, since the coupling mode propagates orthogonally to the two large air holes along with the PS-PCF, when the curvature direction is the same to the axis of two large air holes, the coupling mode experiences a small effective refractive index changes. However, when the curvature direction is perpendicular to the two large air holes, the coupling mode has relatively large effective refractive index change.

\section{Spectral strain characteristic of the LPFG in the PS-PCF}

The strain sensitivity of the resonance peak was observed by fixing one end of the LPFG on a block and the other to a translation stage. The transmission spectra with respect to the induced strain are shown in FIG. 8 (a). The attenuation band has a strain sensitivity of $-0.797 \mathrm{pm} / \mu \varepsilon$.

The axial strain sensitivity of an LPFG may be obtained by differentiating Eq. (1) to yield [10]:

$$
\frac{d \lambda^{m}}{d \varepsilon}=\frac{d \lambda}{d\left(\partial n_{e f f}^{m}\right)}\left(\frac{d n_{\text {core }}}{d \varepsilon}-\frac{d n_{\text {core }}{ }^{m}}{d \varepsilon}\right)+\Lambda \frac{d \lambda^{m}}{d \Lambda}
$$

where the differential refractive index is given by $\partial n_{\text {eff }}=n_{\text {core }}-n_{\text {core }}{ }^{m}$.

In PCFs, as the strain induced along with the LPFG varies, the changes in both the effective reflective indices of the fundamental and second core mode are very similar, by which the first term in Eq. (4) for the material contribution can be ignored. Thus the strain sensitivity strongly depends on the second term in Eq. (4) for the waveguide contribution [14]. Since the slope of the dispersion term, $\frac{d \lambda}{d \Lambda}$ in the PS-PCF is negative, a blue shift was observed as shown in FIG. 8.

\section{Spectral temperature characteristic of the LPFG in the PS-PCF}

The temperature sensitivity was investigated by placing the LPFG upon a hot plate in the range of 30.5 $-82^{\circ} \mathrm{C}$ as shown in FIG. 8. The lowest resolution of the OSA to be used was $0.01 \mathrm{~nm}$, and thereby no change in the center wavelengths of the attenuation band was observed by eye. The temperature sensitivity was estimated to be around $1 \mathrm{pm} /{ }^{\circ} \mathrm{C}$.

The temperature sensitivity of an LPFG can be expressed by differentiating Eq. (1)

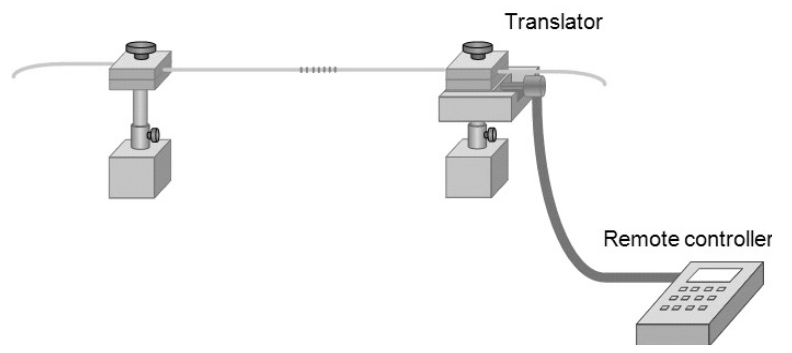

(a)

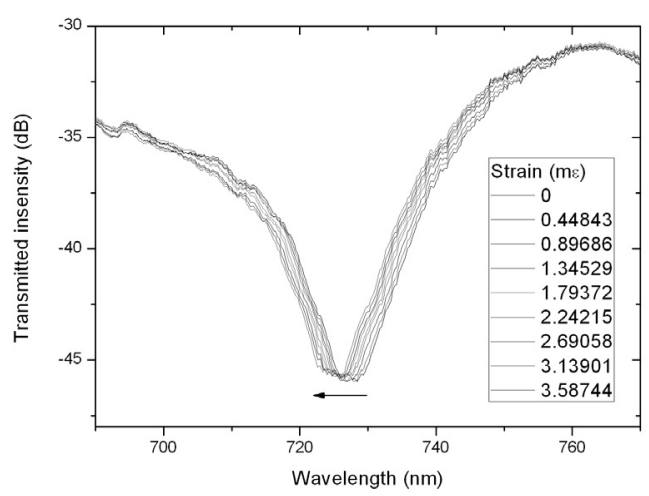

(b)

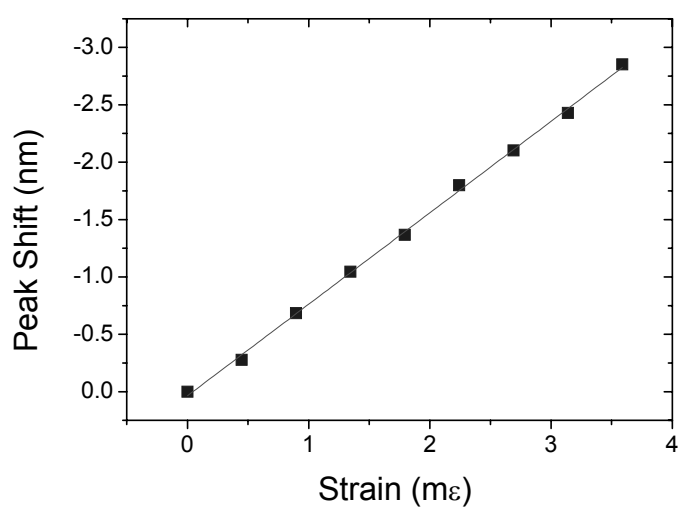

(c)

FIG. 8. (a) Experimental setup for the measurement of strain, (b) Transmission spectra of the LPFG with the strain in range $0-3.6 \mathrm{m \varepsilon}$, (c) wavelength shift as a result of the applied strain.

$$
\frac{d \lambda^{m}}{d T}=\frac{d \lambda}{d\left(\partial n_{\text {eff }}\right)}\left(\frac{d n_{\text {core }}}{d T}-\frac{d n_{\text {corr }}{ }^{m}}{d T}\right)+\Lambda \frac{d \lambda^{m}}{d \Lambda} \frac{1}{L} \frac{d L}{d T}
$$

In conventional fibers such as SMFs, since the core is doped, the effective reflective index of the core mode is more sensitive to temperature than that of the cladding mode, and considerable temperature sensitivity occurs. In PCFs, both the effective reflective indices of the core and higher core mode are similarly dependent on the temperature variation, resulting in the significant reduction in the material contribution given by the first term in Eq. (5). The thermal expansion of PCFs is small 


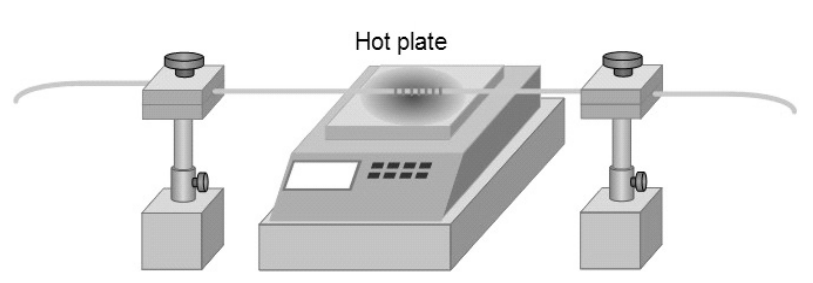

(a)

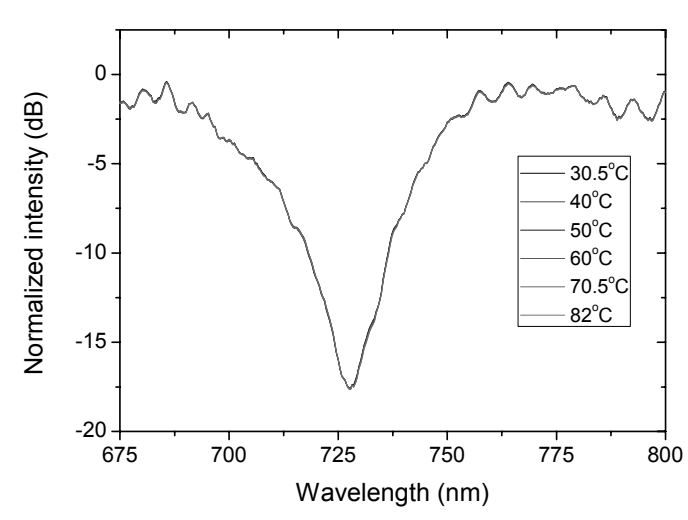

(b)

FIG. 9. (a) Experimental setup for the measurement of temperature, (b) the variation of the resonance wavelength peak as a function of temperature.

enough for the waveguide contribution provided by the second term in Eq. (5) to be ignored, in consequence, PCFs are attractive for measuring various measurands regardless of the effect of temperature as is well-known.

\section{CONCLUSION}

The LPFG inscribed in the PS-PCF with the air discharge method has been investigated for sensitivity to curvature, strain, and temperature. Since the PS-PCF is a multi-mode fiber, the LPFG is attributed to the coupling between the fundamental core mode and the higher core mode. Owing to the asymmetric structure of the PS-PCF with two large air holes outside of a cladding region, the mode was coupled into one mode, an odd mode of LP11. Therefore curvature sensitivity depends on the orientation of the curvature with respect to the axis of the two large air holes, whilst the strain and temperature sensitivity are observed to be similar to LPFGs inscribed in other general PCFs. As the result, the LPFG inscribed in the PS-PCF could be used as a curvature sensor which is sensitive to a desirable axis.

\section{REFERENCES}

1. A. M. Vengsarkar, J. R. Pedrazzani, J. B. Judkins, and P. J. Lemaire "Long-period fiber-grating-based gain equalizers," Opt. Lett. 21, 336-338 (1996).

2. J. K. Bae, J. H. Bae, S. H. Kim, N. K. Park, and S. B. Lee, "Dynamic EDFA gain-flattening filter using two LPFGs with divided coil heaters," IEEE Photon. Technol. Lett. 17, 1226-1228 (2005).

3. V. Bhatia and A. M. Vengsarkar, "Optical fiber long-period grating sensors," Opt. Lett. 21, 692-694 (1996).

4. J. C. Knight, T. A. Birks, P. St. J. Russell, and D. M. Atkin, "All-silica single-mode optical fiber with photonic crystal cladding," Opt. Lett. 21, 1547-1549 (1996).

5. H. Dobb, K. Kalli, and D. J. Webb, "Measured sensitivity of arc-induced long-period grating sensors in photonic crystal fibre," Opt. Comm. 260, 184-191 (2005).

6. G. Kakarantzas, T. A. Birks, and P. St. J. Russell, "Structural long-period gratings in photonic crystal fibers," Opt. Lett. 27, 1013-1015 (2002).

7. G. Brambilla, A. A. Fotiadi, S. A. Slattery, and D. N. Nikogosyan, "Two-photon photochemical long-period grating fabrication in pure-fused-silica photonic crystal fiber," Opt. Lett. 31, 2675-2677 (2006).

8. J. H. Lim, K. S. Lee, J. C. Kim, and B. H. Lee, "Tunable fiber gratings fabricated in photonic crystal fiber by use of mechanical pressure," Opt. Lett. 29, 331-333 (2004).

9. G. Humbert, A. Malki, S. Février, P. Roy, and D. Pagnoux, "Electric arc-induced long-period gratings in Ge-free air-silica microstructure fibres," Electron. Lett. 39, 349-350 (2003).

10. T. Cho, G. Kim, K. Lee, J. M. Jeong, and S. B. Lee, "Study on the fabrication process of polarization maintaining photonic crystal fibers and their optical properties," J. Opt. Soc. Korea 12, 19-24 (2008).

11. G. Kim, T. Cho, K. Hwang, K. Lee, K. S. Lee, and S. B. Lee, "Control of hollow-core photonic bandgap fiber ellipticity by induced lateral tension," Opt. Exp. 17, 1268-1273 (2009).

12. S. D. Lim, H. C. Park, I. K. Hwang, and B. Y. Kim, "Combined effects of optical and acoustic birefringence on acousto-optic mode coupling in photonic crystal fiber," Opt. Exp. 16, 6125-6133 (2008).

13. N. H. Vu, I. K. Hwang, and Y. H. Lee, "Bending loss analyses of photonic crystal fibers based on the finite-difference time-domain method," Opt. Lett. 33, 119-121 (2008).

14. Y. G. Han, G. Kim, K. Lee, S. B. Lee, C. H. Jeong, C. H. Oh, and H. J. Kang, "Bending sensitivity of long-period fiber gratings inscribed in holey fibers depending on an axial rotation angle," Opt. Exp. 15, 12866-12871 (2007). 